\title{
Real-Time Selective Harmonic Minimization in Cascaded Multilevel Inverters with Varying DC Sources
}

\author{
F. J. T. Filho*, T. H. A. Mateus ${ }^{* *}$, H. Z. Maia**, B. Ozpineci***, J. O. P. Pinto** and L. M. Tolbert* \\ * The University of Tennessee/Department of Electrical Engineering, Knoxville, USA \\ ${ }^{* *}$ Federal University of Mato Grosso do Sul /Department of Electrical Engineering, Campo Grande, Brazil \\ ${ }^{* * *}$ Oak Ridge National Laboratory, Knoxville, USA
}

\begin{abstract}
A new approach for selective harmonic elimination in a 7-level cascaded multilevel inverter with separate DC sources will be presented. As opposed to previous research in this area, the DC sources feeding the multilevel inverter are considered to be varying in time. This method uses genetic algorithms to obtain switching angles offline for different DC source values and uses neural networks to determine the switching angles that correspond to the real-time values of the DC sources. This implies that each one of the DC sources of this topology can have different values at any time but the output fundamental voltage will stay constant and the harmonic will still meet the specifications. The paper gives details on the approach used, together with simulation and experimental results.
\end{abstract}

\section{INTRODUCTION}

There have been many technical papers using selective harmonic elimination or minimization for the fundamental frequency operation of the cascaded multilevel inverters with separate DC sources. In [1], genetic algorithms have been used to determine the optimal switching angles for DC sources of equal values. Analytical solutions for this problem using the theory of symmetric polynomials were also reported for unipolar and bipolar schemes [2-3]. All of these papers assumed that the DC sources are equal and do not vary with time. In [4] and [5], analytical solutions for the case of unequal DC sources have been derived. All of these papers use computationally intensive time consuming equations to solve for the angles; therefore, the switching angles are calculated off-line.

The authors of [6-7] have developed methods to calculate the switching angles in real-time; however, their approach was not extended for unequal DC sources.

An alternate approach to determining the optimum switching angles in real-time for varying DC sources is to calculate the switching angle solutions off-line using one of the methods listed above and storing the solutions in a look-up table. For accurate representation of every solution for every different DC source case would require a huge look-up table. Even then, for some situations, the solutions might be missing and some kind of interpolation would be required. In this paper, the look up table is replaced by artificial neural networks (ANN) which, if trained well, have the inherent capability of generalizing solutions. What this means is that, if the correct range of data are used for training and if the ANN is not over trained, the network will fill in the solution gaps properly.
Since ANN runs fast, it is possible to quickly determine the switching angles to realize real-time time control.

\section{PROBLEM DEFINITION}

In a practical case, it is necessary to consider the variation of the DC sources along with its operation as in a solar panel. This means that during a day of operation, the solar panel output voltage may vary according to the amount of energy available, and the system must give a response to this variation in the switching angles to keep the fundamental regulated to its reference value and the low order harmonics minimized. One approach for this problem of unequal DC sources was presented in [4-5] using symmetric polynomials where all the solutions (switching angles) are defined where there is a solution.

The proposed approach in this work will be done in two steps. First a Genetic Algorithm (GA) will be implemented to find the switching angles for a determined set of pre-determined input voltages.

An important feature of GA for this approach is that for the range space where no analytical solution exists the GA will find the nearest solution providing a smooth data set that is desired for the neural network training. Then, with the previous data set a neural network will be trained to give the set of angles for each voltage situation.

\section{A. Genetic Algorithms}

Genetic Algorithm (GA) is a technique based on the evolutionary process. It mimics the behavior of populations during generations based on the idea that through consecutive generations the best individuals have more probability to survive to pass its genetic code, the information, by action of biological operators for its descendants. Through generations, the quality of the population has a trend to improve. The obtained optimum is an end product containing the best elements of previous generations where the attributes of a stronger individual tend to be carried forward into the following generation [8-9].

A GA is a stochastic search method that has been shown to be well suited for problems where there are many global minimum and/or highly dimensional search spaces. Each individual of a set has an associated cost value, called fitness function that is a measure of how good this individual is in the population. In the previous work analytical solutions were found partially in the range space of input voltage variation, however this approach 


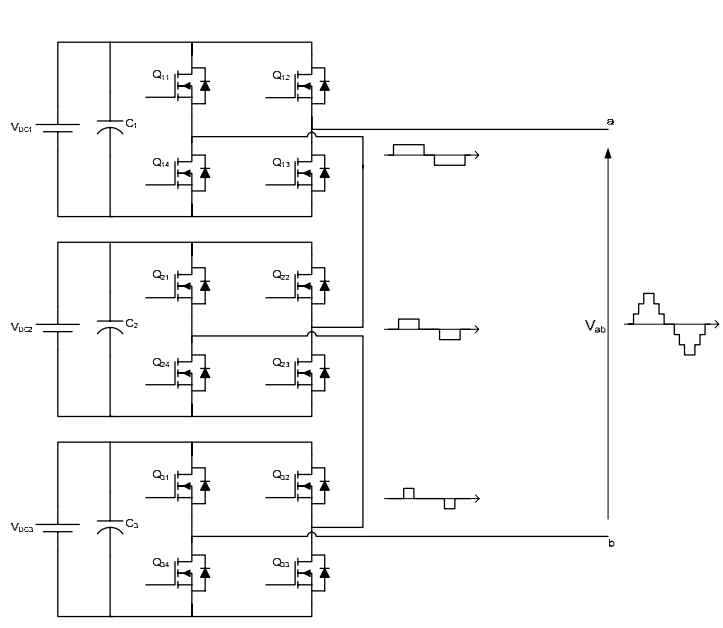

(a)

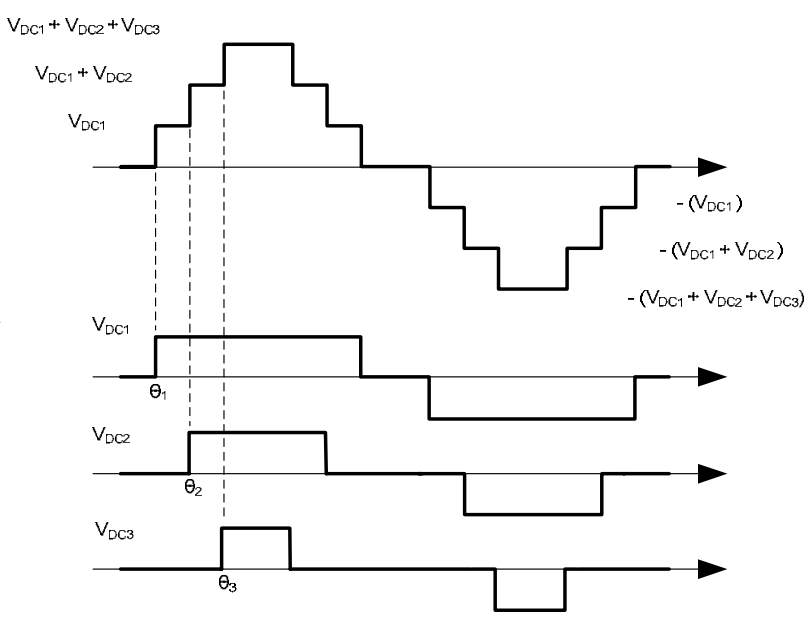

(b)

Fig. 1. Cascaded multilevel inverter: (a) topology and (b) voltage output waveform generation.

still uses GA first because this range is used to calibrate it to perform in the range where there is no analytical solution. Therefore it is found the correct GA parameters to bias the algorithm through the desired solution. Second, it is possible to adjust the algorithm to perform its solutions looking at the previous results giving a future solution, or angles in this case, which are smooth so that the ANN can be easily trained.

In a practical situation in a multilevel converter, all the DC sources vary to some degree. This variation can be proportional to the state of charge as it is in a battery or fuel cell system or it can be a function of solar irradiation as in a solar panel. In this way it is necessary to control the switching angles to keep the desired output voltage characteristics. The output voltage waveform shown in Fig. 1(b) can be expressed in the Fourier form as:

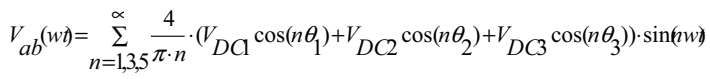

where, $V_{D C 1}, V_{D C 2}, V_{D C 3}=$ input DC sources

$$
\begin{aligned}
& \theta_{1}, \theta_{2}, \theta_{3}=\text { inverter switching angles } \\
& V_{a b}=\text { inverter output voltage }
\end{aligned}
$$

Equation (1) shows the contents of the output voltage at infinite frequencies (odd harmonic content). Under variations in the DC input sources, it is desired to maintain the fundamental output voltage and cancel the lowest nontriple harmonics, in this case the $5^{\text {th }}$ and $7^{\text {th }}$. In applications for three phase machine drive, there is no need to cancel the harmonics multiple of three because it is cancelled in the line voltage. The set of equation for the GA are:

$$
\begin{aligned}
& V_{f u n d}=\frac{4}{\pi} \cdot\left(V_{D C 1} \cos \left(\theta_{1}\right)+V_{D C 2} \cos \left(\theta_{2}\right)+V_{D C 3} \cos \left(\theta_{3}\right)\right) \\
& V_{5 t h}=\frac{4}{\pi \cdot 5} \cdot\left(V_{D C 1} \cos \left(\theta_{1}\right)+V_{D C 2} \cos \left(\theta_{2}\right)+V_{D C 3} \cos \left(\theta_{3}\right)\right) \\
& V_{7 t h}=\frac{4}{\pi \cdot 7} \cdot\left(V_{D C 1} \cos \left(\theta_{1}\right)+V_{D C 2} \cos \left(\theta_{2}\right)+V_{D C 3} \cos \left(\theta_{3}\right)\right)
\end{aligned}
$$

Equations (2), (3) and (4) are used by the GA to obtain the data set of angles to control the multilevel inverter for each value of the DC sources. Rewriting the equations above to satisfy the problem requirements:

$$
\begin{gathered}
110 \sqrt{2} \pi / 4-V_{D C 1} \cos \left(\theta_{1}\right)-V_{D C 2} \cos \left(\theta_{2}\right)-V_{D C 3} \cos \left(\theta_{3}\right)=0 \\
V_{D C 1} \cos \left(5 \theta_{1}\right)+V_{D C 2} \cos \left(5 \theta_{2}\right)+V_{D C 3} \cos \left(5 \theta_{3}\right)=0 \\
V_{D C 1} \cos \left(7 \theta_{1}\right)+V_{D C 2} \cos \left(7 \theta_{2}\right)+V_{D C 3} \cos \left(7 \theta_{3}\right)=0
\end{gathered}
$$

To solve (5), (6) and (7) using GA, it is necessary to provide the real DC source values and the desired output voltage. The output voltage is intended to be equal to 110 $\mathrm{V}_{\mathrm{ac}}$ as shown in (5) and the data set is solved based on the value of the three input DC sources provided. After measuring the real values of the DC sources, a set of angles is found so that the output voltage is kept constant and the fifth and seventh harmonics are eliminated. An objective function that represents grades, evaluates and classifies each individual in the population was defined as follows:

$$
f\left(V_{\text {fund }}, V_{5 t h}, V_{7 t h}\right)=k_{1}\left|V_{\text {fund }}-110\right|+k_{2}\left|V_{5 t h}\right|+k_{3}\left|V_{7 t h}\right|
$$

In Eq. (8) the coefficients $k_{2}$ and $k_{3}$ need to have higher value than $\mathrm{k} 1$ at the same time that it must allow the GA to do a non biased optimization. Fig. 2 shows the GA objective function under variation of the three DC input sources. In the $\mathrm{x}$ axis is each combination of the input

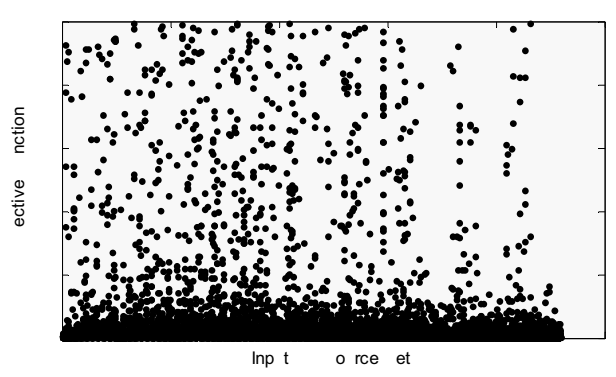

Fig. 2. GA results under DC input variation. 
sources .

The solution of (8) requires that for a fixed voltage in two input voltages requires a variation in all range for the third one. This a combinatorial problem since there is not previous information about the maximum difference allowed between the DC sources. The resolution of the DC input sources for the data set will define the size of the data set for training of the neural network. If a range from 50 to 90 Volts is defined for each DC source with step of one volt for the first source, it will be forty-one points for each of three sources leading to a data set of size $41^{3}$. This result in a data set of size 68921 . To avoid such a large data set, the DC sources are defined to vary from 50 to 90 Volts with step of 4 Volts leading to a data set of size 1331. In Table I a partial view of this data set is presented.

\section{B. Artificial Neural Network}

An important feature of Artificial Neural Network (ANN) that made it suitable for this problem is its flexibility to lead in its domain and outside it [10]. Although the data set presented to the ANN is not complete and not all combinations were obtained by the GA, the ANN has flexibility enough to interpolate and extrapolate the results. The data set in Table I was used to train the neural network.

The ANN topology proposed is presented in Fig. 3. It is a feedfoward ANN with a tangent-sigmoid function activation hidden layer and a linear activation function output layer. Different feedforward topologies were taken under different training methods to investigate which one fits for this application. This ANN takes the real DC source values and gives the switching angles for the control system.

The ANN simulation results can be seen in Fig. 4. Three situations are presented in these figures through the variation of one of the three DC sources while keeping the other two at a constant value. In the first third of the graph, it was made the variation of the upper DC source while the other two lower sources are kept constant. The angles variation under these conditions found by the GA and shown in Fig. 4 for the ANN output are very smooth. Under this condition, the output was kept in its nominal voltage with no more than $1 \mathrm{~V}$ of deviation. The harmonics level of individual $5^{\text {th }}$ and $7^{\text {th }}$ harmonics are kept at very low values as shown in Fig. 4(b).

TABLE I.

DATASET OBTAINED THROUGH GA RUN

\begin{tabular}{|c|c|c|c|c|c|}
\hline $\begin{array}{c}\mathrm{V}_{\mathrm{dc} 1} \\
(\mathrm{~V})\end{array}$ & $\begin{array}{c}\mathrm{V}_{\mathrm{dc} 2} \\
(\mathrm{~V})\end{array}$ & $\begin{array}{c}\mathrm{V}_{\mathrm{dc} 3} \\
(\mathrm{~V})\end{array}$ & $\begin{array}{c}\theta_{1} \\
(\text { Degrees })\end{array}$ & $\begin{array}{c}\theta_{2} \\
(\text { Degrees })\end{array}$ & $\begin{array}{c}\theta_{3} \\
(\text { Degrees })\end{array}$ \\
\hline 52 & 52 & 52 & 11.7 & 31.5 & 58.7 \\
\hline 52 & 52 & 56 & 12.6 & 33.4 & 59.8 \\
\hline.. &.. &.. &.. &.. &.$\cdot$ \\
\hline 52 & 52 & 92 & 27.7 & 46.9 & 63.7 \\
\hline 52 & 56 & 52 & 12.0 & 34.6 & 60.9 \\
\hline 52 & 56 & 56 & 13.2 & 36.4 & 61.7 \\
\hline.. &.. &.. &.. &.. &.. \\
\hline 52 & 56 & 92 & 29.7 & 47.6 & 64.6 \\
\hline
\end{tabular}

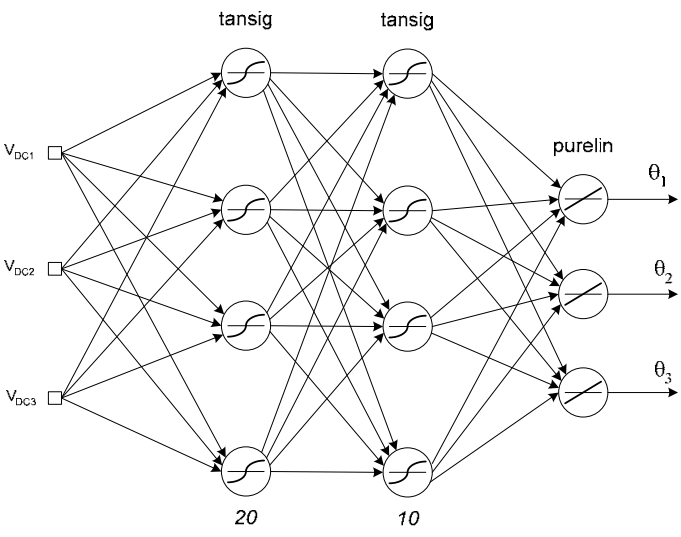

Fig. 3. Feed forward artificial neural network topology.

The seven level output waveform simulation using the Feed forward ANN trained is shown in Fig. 5 for a random value chosen for the DC input voltages. This ANN updates the angles during each cycle of the fundamental frequency. The frequency spectrum of the output voltage is shown in Fig. 6 for a cycle of the fundamental frequency. It can be seen that the $5^{\text {th }}$ and $7^{\text {th }}$ harmonics are greatly minimized using the angles provided by the ANN. An analysis over the outputs of the neural network shows that in the worst case situations the harmonics do not go beyond $1 \%$ of the fundamental output voltage.

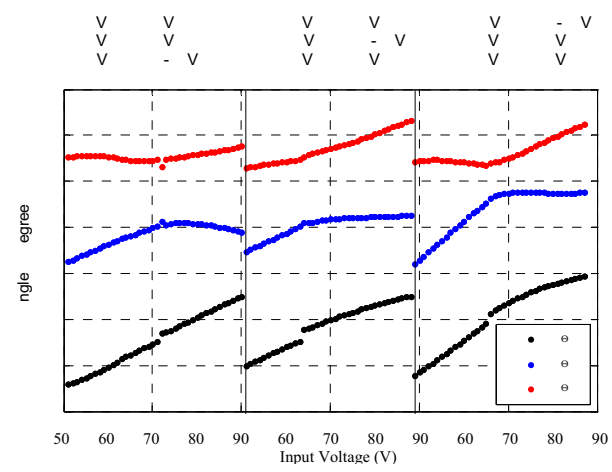

(a)

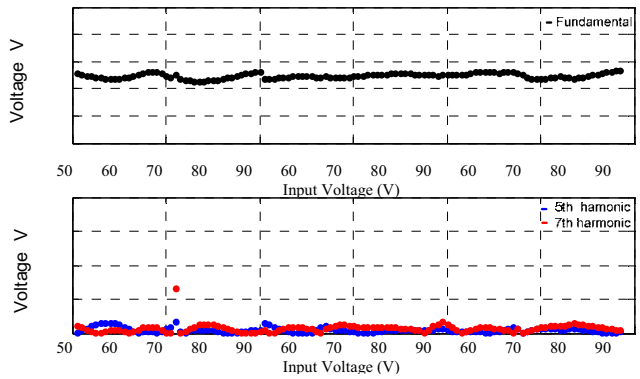

(b)

Fig. 4. (a) Neural network angles output under DC input voltage variation and (b) voltage output characteristics. 


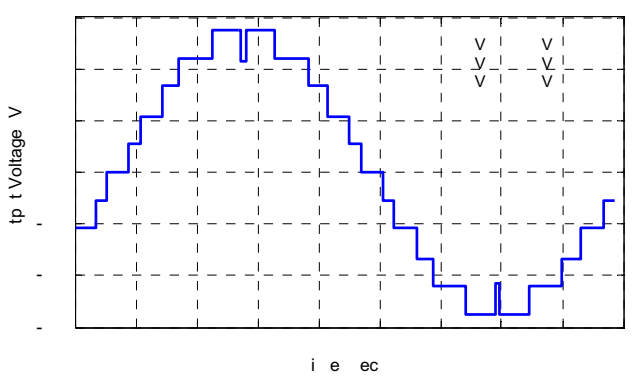

Fig. 5. Line output voltage waveform.

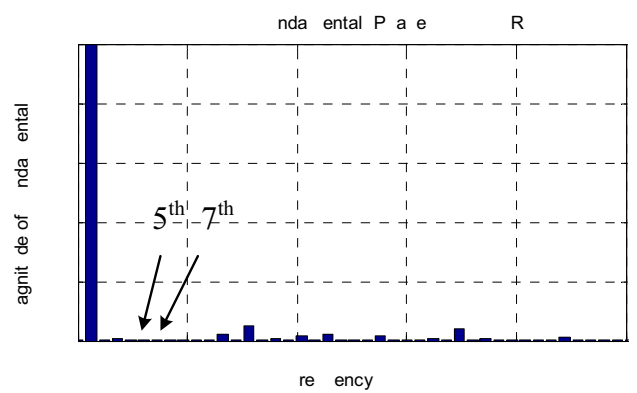

Fig. 6. Output voltage frequency spectrum.

\section{AG Exploration of the Search Space}

The set of equations (5), (6), and (7) does not have solutions for all the range, which means that at some input voltages there are no solutions to satisfy the criterion desired for the fundamental and to completely cancel the low order harmonics. However, in this case the GA will look for an approximate solution that is near to the requirement. This will introduce a set of solutions that partially satisfy the set of equations (5), (6) and (7). This procedure will allow the fundamental to be around its nominal value $(5 \%)$ and/or a low order harmonic that is not canceled but instead is very low. This characteristic is very important for the neural network training process and is shown in Fig. 7 when only one of the DC sources are varying. Beginning at $39 \mathrm{~V}$, the GA cannot find a solution because none exists in that range. It is possible to see in the figure an increase in the output voltage caused by the approximated solution found. In this case the $5^{\text {th }}$ and $7^{\text {th }}$ harmonics are minimized to less than $1 \%$ with the output voltage around its nominal value.

\section{Neural Network Real Time Control System IMPLEMENTATION}

The control system was implemented using RTLAB, a real-time computing platform [12]. A computer with two processors, an IP341 module for analog-todigital conversion and an IP470 for digital output was the hardware target. The first processor is responsible for the acquisition of the DC source voltages fed to the ANN that performs the calculation of the appropriate switching angles. The second processor receives the angles from the master processor and generates the corresponding pulses. The step size employed in the computation was $100 \mu \mathrm{s}$.

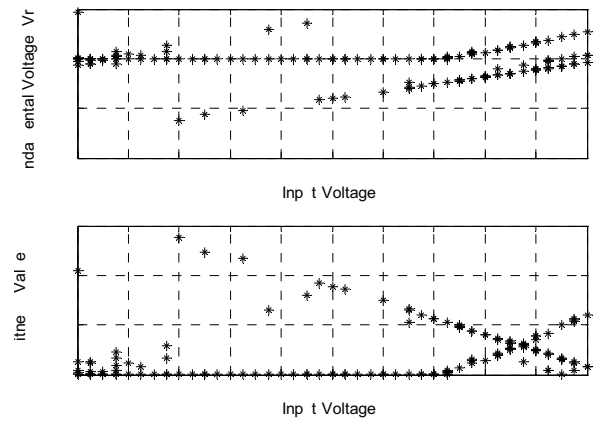

Fig. 7. Results when only one the DC source is varying.

Because the modulation is software synchronized there is a little fluctuation in the command pulses width even when there is no variation in the input DC voltages. This is due to the imprecision associated with a software timer that exists even for a real-time operating system as the one running at the target computer. That does not compromise the stability of the overall system and has little impact on the harmonic elimination performance.

\section{EXPERIMENTAL RESULTS}

The multilevel inverter prototype is shown in Fig. 8. It is a three phase 11-level H-Bridge.

In Fig. 9 the experimental results for a seven level inverter operating with unequal DC sources are shown with the voltage values indicated. In Fig. 10 the frequency spectrum is shown where it can be noticed that the target harmonics were minimized. In this same figure it can be noticed a high value of the $3^{\text {rd }}$ harmonic, this harmonic was not minimized due to the fact that it will be canceled in line voltage for a three phase application.

This system has the ability to update the angles in real time at speeds higher than the low switching frequency but the angle speed update is done at the end of a cycle of the low frequency to avoid even harmonics. It is not assumed a substantial step variation in the magnitude of the DC source inputs for this approach, but the system is still able to respond to this variation promptly after one cycle of the fundamental and not during the step change. In Figs. 11 and 12, another case is shown with less

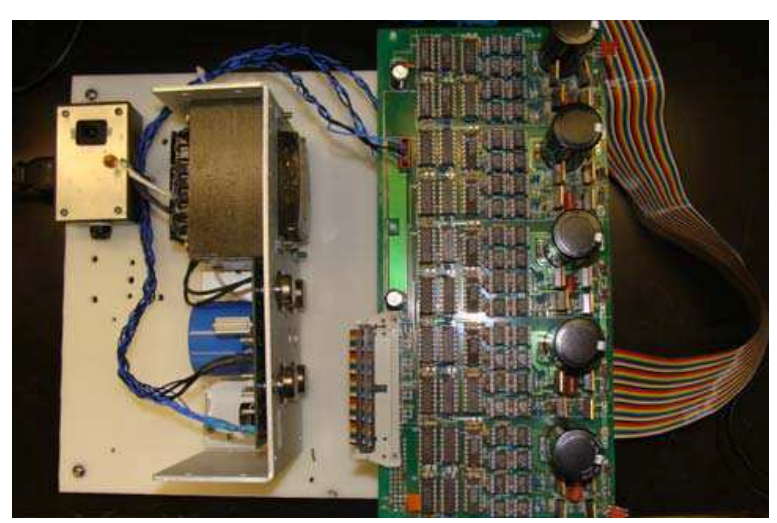

Fig. 8. Experimental cascaded H-bridge converter. 


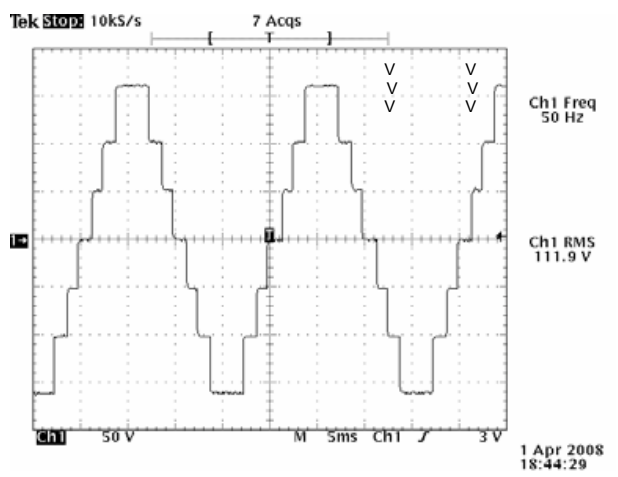

Fig. 9. Output voltage waveform.

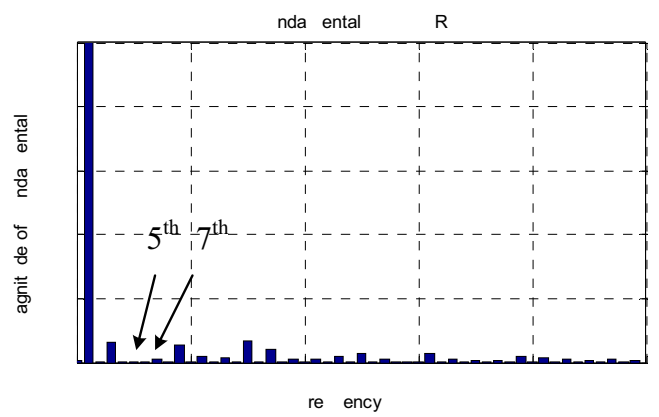

Fig. 10. Frequency spectrum of output voltage waveform.

variation between the DC input sources showing that the output of the ANN is still accurate enough to minimize the low order harmonics presented.

\section{CONCLUSIONS}

A new approach for real time computation of switching angles using artificial neural networks was presented. The solutions were found off line using genetic algorithms to obtain a data set for use during the training process of the neural network and to explore the advantages of approximate solutions obtained by the GA. The trained neural network is used then for on-line real time determination of the angles.

Experimental results were shown to validate this approach. A real time system was implemented using the neural network previously obtained. The angles are updated at the same frequency of the fundamental output voltage due to the low computation required for the neural network.

Future works include the behavior of the neural network under a larger number of angles for minimization of non triplens harmonics and a greater number of DC input sources in three phase applications.

\section{REFERENCES}

[1] B. Ozpineci, L. M. Tolbert, J. N. Chiasson, "Harmonic optimization of multilevel converters using Genetic Algorithms," IEEE Power Electronics Letters, vol.3, no.3, pp. 92-95, Sept. 2005.

[2] J. N. Chiasson, L. M. Tolbert, K. J. McKenzie, Z. Du, “A unified approach to solving the harmonic elimination equations in multilevel converters," IEEE Transactions on Power Electronics, March 2004, vol. 19, no. 2, pp. 478-490.

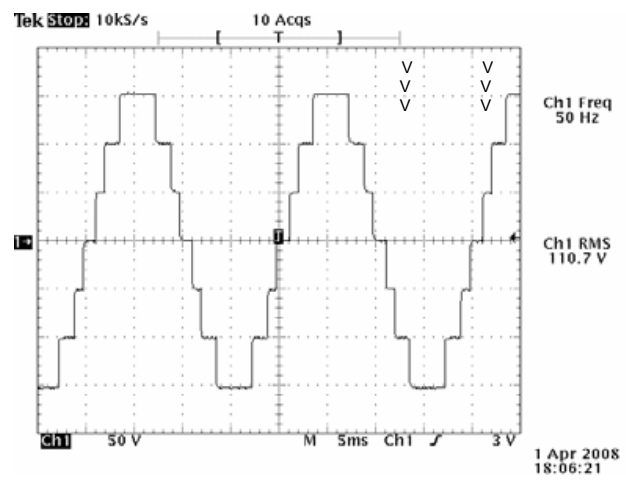

Figure 11. Output voltage waveform

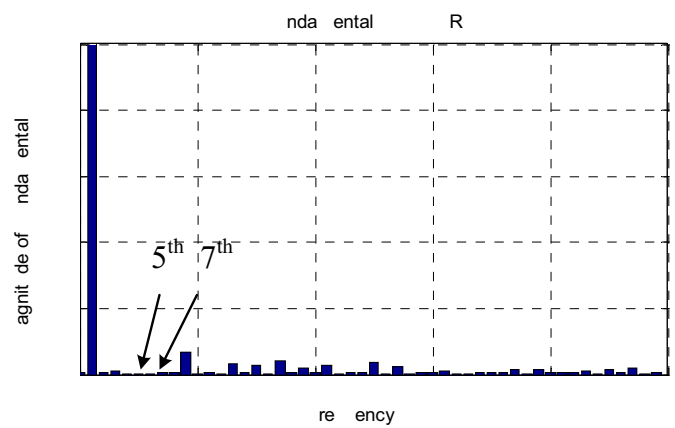

Fig. 12. Frequency spectrum of output voltage waveform.

[3] J. N. Chiasson, L. M. Tolbert, K. J. McKenzie, Z. Du, "Elimination of harmonics in a multilevel converter using the theory of symmetric polynomials and resultants," IEEE Transactions on Control Systems Technology, vol. 13, no. 2, March 2005, pp. 216-223.

[4] Zhong Du, L. M. Tolbert, J. N. Chiasson, and Hui Li, "Low switching frequency active harmonic elimination in multilevel converters with unequal DC voltages," Annual Meeting of the IEEE Industry Applications Society, vol.1, pp. 92-98 Vol. 1, 2-6 Oct. 2005.

[5] Z. Du, L. M. Tolbert, J. N. Chiasson, "Active harmonic elimination for multilevel converters," IEEE Transactions on Power Electronics, vol.21, no.2, pp. 459-469, March 2006.

[6] D. W. Kang, H. C. Kim, T. J. Kim, and D. S. Hyun, "A simple method for acquiring the conducting angle in a multilevel cascaded inverter using step pulse waves," IEE Proceedings on Electric Power Applications, vol. 152, pp. 103-111, 2005.

[7] Y. Liu, H. Hong, A. Q. Huang, "Real-time calculation of switching angles minimizing THD for multilevel inverters with step modulation," IEEE Transactions on Industrial Electronics, in press.

[8] D. E. Goldberg, "Genetic Algorithms in Search, Optimization, and Machine Learning”. Reading, MA: Addison-Wesley, 1989.

[9] K.F. Man, K.S. Tang, S. Kwong, "Genetic algorithms: concepts and applications [in engineering design]," IEEE Transactions on Industrial Electronics, vol.43, no.5, pp.519-534, Oct 1996.

[10] A.K. Jain, Jianchang Mao and K.M. Mohiuddin, "Artificial neural networks: a tutorial," IEEE Computer Magazine, vol.29, no.3, pp.31-44, Mar 1996

[11] RT-LAB, Opal-RT Technologies (2001): http://www.opal-rt.com. 